\title{
Fetomaternal outcomes in conservative management of preterm premature rupture of membrane
}

\author{
Rama Chundawat, Meenakshi Singh*, Arun Gupta, Bhamini Jakhetiya, Ruhina Khan
}

Department of Obstetrics and Gynecology, Geetanjali Medical College and Hospital, Udaipur, Rajasthan, India

Received: 16 December 2020

Accepted: 18 January 2021

\section{*Correspondence:}

Dr. Meenakshi Singh,

E-mail: meenaxiposwal@gmail.com

Copyright: (c) the author(s), publisher and licensee Medip Academy. This is an open-access article distributed under the terms of the Creative Commons Attribution Non-Commercial License, which permits unrestricted non-commercial use, distribution, and reproduction in any medium, provided the original work is properly cited.

\begin{abstract}
Background: The management of PPROM remains controversial. Aim of this study was to determine the fetomaternal outcome in conservative management of PPROM.

Methods: A prospective observational study was done on a total of 110 pregnant women between 24 and 36 weeks 6 days gestation with PPROM visiting Geetanjali medical college and hospital from February 2019 to July 2020 . All the patients were subjected to detailed history and examination and followed till their delivery.

Results: The incidence of PPROM in our study was $4.94 \%$. The mean latency period from membrane rupture to delivery decreased from $12.1 \pm 14$ days at $>28$ weeks, $5.3 \pm 7.7$ days at 28-31 weeks 6 days to $1.1 \pm 1.2$ days at 32 to 36 weeks 6 days. The most common indication of LSCS was fetal distress (26.31\%). E. coli was the most common organism $(36.36 \%)$ isolated and it was significantly associated with gestational age at PPROM (p value <0.040405). The maternal morbidity was $24.53 \%$ and was closely associated with the gestational age at PPROM (p value $<0.001$ ). The perinatal morbidity was $44.20 \%$ and perinatal mortality was $13.04 \%$ which was mainly due to RDS (33.34\%).

Conclusions: Management of PPROM involves complete evaluation of risks and benefits of conservative management. The treatment should be directed toward conserving the pregnancy with prophylactic use of antibiotics and corticosteroids thereby reducing fetomaternal morbidity and mortality. However, termination of pregnancy should be considered at the earliest suspicion of chorioamnionitis, septicaemia.
\end{abstract}

Keywords: Chorioamnionitis, Conservative management, Fetomaternal outcome, Preterm PROM

\section{INTRODUCTION}

PPROM is defined as rupture of fetal membrane with leakage of the amniotic fluid before onset of regular uterine contractions before 37 weeks of gestation. ${ }^{1,2}$ It is one of the most common cause of prematurity contributing to 30 to $40 \%$ of preterm births. ${ }^{3}$

The conservative management of PPROM, with use of prophylactic antibiotics and steroids has led to improvement in the maternal-fetal outcome. However, neonatal outcome largely depends upon the neonatal care facilities. This study was done with the aim to find out the fetomaternal outcome in conservative management of PPROM.

\section{METHODS}

It was a prospective observational study. The study was conducted in department of Obstetrics and Gynaecology, Geetanjali Medical College and Hospital, Udaipur, Rajasthan for the period of 1.5 year from February 2019 to July 2020 after approval form Institutional Research Review Committee and Institutional Ethical Committee.

\section{Source of data}

All pregnant mothers who were admitted with PPROM in department of Obstetrics and Gynaecology, Geetanjali Medical College and Hospital, Udaipur, Rajasthan. 


\section{Sampling technique}

Purposive Consecutive Sampling was used in this study.

\section{Minimum sample size}

Minimum sample size was 110 patients admitted with PPROM.

\section{Inclusion criteria}

All pregnant women between 24-36 weeks 6 days of gestational age with preterm premature rupture of membranes.

\section{Exclusion criteria}

Fetal anomalies, chronic liver disease, class II to IV cardiac diseases, chronic renal failure, patients who refuse to participate in the study.

A detailed history was taken including age of patients, booking, socio-economic status, parity, menstrual history, time of rupture of fetal membrane, amount of amniotic fluid lost, and its colour, association with pain or bleeding per vagina and perception of fetal movements, history of PPROM in previous pregnancies and history suggestive of cervical incompetence, history of P/V done before admission were noted in a prefilled proforma. In all cases, routine haematological investigations, urine examination, cardiotocography and obstetric ultrasonography was performed. General physical and obstetric examination was done. Sterile speculum examination was done to confirm the diagnosis of leaking per vagina. When in doubt litmus paper test was done to confirm leaking. The ultrasound finding of reduced amniotic fluid further confirmed the diagnosis. Digital examination was done only in cases with suspicion of pre-term labour. High vaginal swab was taken. All the patients were managed conservatively with antibiotics as per culture sensitivity report and corticosteroid. The patients were monitored clinically for signs of chorioamnionitis. Serial ultrasound was done to monitor the amount of liquor. TLC/DLC was done twice weekly and high vaginal swab was sent weekly. Pregnancy was terminated irrespective of gestation, in women with clinical chorioamnionitis, non-reassuring fetal heart, and fetal death. If infection was identified, delivery was expedited and antibiotic stepped up.

The labour was allowed to progress either spontaneously or induced either by intra cervical instillation of PGE2 gel or continuous infusion of oxytocin depending on the bishops score and monitored carefully by partograph. Caesarean section was done for obstetric indication. The patients were monitored for third stage complications like PPH and retained placenta and followed up in puerperal period. Neonates were closely observed for any evidence of neonatal sepsis and other complications like respiratory distress syndrome (RDS), necrotizing enterocolitis, intraventricular haemorrhage in NICU. Neonatal morbidity and mortality were noted. The neonatal sepsis was confirmed by blood culture and sensitivity. Both mother and neonates were followed up till their stay in the hospital.

\section{Statistical analysis}

All the results were expressed in mean $\pm \mathrm{SD}$ or frequency (percentage). Statistical analysis was done by SPSS software 26 version. $\mathrm{P}$ value $<0.05$ was considered as significant.

\section{RESULTS}

The total number of deliveries conducted during the study period was 2224 out of these 110 patients presented with PPROM. The incidence of PPROM was $4.94 \%$. The mean age of the women was $30.05 \pm 5.5$ years $(20-48)$. Majority of the women $(49.09 \%)$ in our study came from $\mathrm{V}$ class of the society and $32.73 \%$ belonged to IV class of society according to modified Kuppuswamy scale. In our study, most of cases were booked $(81.82 \%)$. The number of twin pregnancies were $28(25.45 \%)$. More than $50.91 \%$ were multigravida.

Table 1: Distribution of women according to gestational age at PPROM and latency period with respect to gestational age at delivery.

\begin{tabular}{|lllll|}
\hline $\begin{array}{l}\text { Gestational age at } \\
\text { admission (in weeks) }\end{array}$ & $\begin{array}{l}\text { Number of } \\
\text { patients }(\mathbf{n})\end{array}$ & $\begin{array}{l}\text { Mean gestational age at } \\
\text { admission (in weeks) }\end{array}$ & $\begin{array}{l}\text { Mean gestational age at the } \\
\text { time of delivery (in weeks) }\end{array}$ & $\begin{array}{l}\text { Latency periods } \\
\text { (days) (mean } \pm \text { SD) }\end{array}$ \\
\hline <28 weeks & $10(9.09 \%)$ & $26.7 \pm 0.73$ & $27.7 \pm 1.79$ & $12.1 \pm 14$ days \\
\hline $\mathbf{2 8 - 3 1 w e e k s ~ 6 ~ d a y s ~}$ & $24(21.82 \%)$ & $29.32 \pm 1.17$ & $29.9 \pm 1.81$ & $5.3 \pm 7.7$ days \\
\hline 32-36weeks 6 days & $76(69.09 \%)$ & $33.81 \pm 1.21$ & $33.96 \pm 1.17$ & $1.1 \pm 1.2$ days \\
\hline
\end{tabular}

In the present study, women with PPROM with gestational age $>31$ weeks 6 -day, only $11.84 \%$ cases had latent phase of $>72$ hours, while $81.57 \%$ of women delivered within 24 hours of rupture membrane ( $\mathrm{p}$ value was $<0.00001)$. The mean latency period from membrane rupture to delivery decreased from $12.1 \pm 14$ days at $>28$ weeks, $5.3 \pm 7.7$ days at $28-31$ weeks 6 days to $1.1 \pm 1.2$ days at 32 to 36 weeks 6 days. The gestational age at presentation in majority of the women was between 32 and 36 week 6 days $(69.09 \%)$ (Table 1$)$. 
Table 2: Amniotic fluid culture in relation to gestational age.

\begin{tabular}{|llll|}
\hline $\begin{array}{llll}\text { Amniotic fluid } \\
\text { culture growth }\end{array}$ & \multicolumn{2}{l|}{ Gestational age (weeks) } & \\
\hline E. coli & $3(30 \%)$ & $8(33.33 \%)$ & $29(38.15 \%)$ \\
\hline Klebsiella & $2(20 \%)$ & $7(29.16 \%)$ & $11(14.47 \%)$ \\
\hline Pseudomonas & $2(20 \%)$ & $3(12.5 \%)$ & $1(1.31 \%)$ \\
\hline Sterile & $3(30 \%)$ & $6(25 \%)$ & $35(46.05 \%)$ \\
\hline
\end{tabular}

On analysing the results of high vaginal swab culture taken prior to initiation of antibiotics, it was observed that in $60 \%$ the culture was positive. The most common organism grown was Escherichia coli $(36.36 \%)$, followed by Klebsiella (18.18\%), Pseudomonas aeruginosa $(5.45 \%)$. Among <28 weeks gestation age group $30 \%$ high vaginal swab was positive while in 32-36 weeks gestation age group $(38.15 \%)$. E. coli was the most common organism irrespective to gestation age ( $\mathrm{p}$ value was 0.040405) (Table 2).

The most common risk factor for PPROM in our study was Twins $(24.54 \%)$ followed by cervical incompetence (9.90\%), malpresentation $(5.45 \%)$, patients with previous history of PPROM (2.72\%), urogenital tract infection $(2.72 \%)$, elderly gravida $(1.81 \%)$, abruptio placentae $(1.81 \%)$ bicornuate uterus $(0.90 \%)$.

Table 3: Maternal morbidity in relation to latency period.

\begin{tabular}{|llll|}
\hline Maternal morbidity $(\mathbf{n}=\mathbf{2 7})$ & Latency period & & >72 hours $(\mathbf{n}=7)$ \\
\hline Clinical chorioamnionitis & $\mathbf{0 - 2 4}$ hours $(\mathbf{n = 1 3})$ & $\mathbf{2 4 - 7 2}$ hours $(\mathbf{n = 7})$ & $4(57.14 \%)$ \\
\hline Abruptio placenta & $0(0 \%)$ & $2(28.57 \%)$ & $1(14.28 \%)$ \\
\hline Wound infection & $4(30.76 \%)$ & $2(28.57 \%)$ & $1(14.28 \%)$ \\
\hline Retained placenta & $1(7.69 \%)$ & $0(0 \%)$ & $0(0 \%)$ \\
\hline Puerperal pyrexia & $3(23.076 \%)$ & $1(14.28 \%)$ & $1(14.28 \%)$ \\
\hline
\end{tabular}

Table 4: Maternal morbidity in relation to gestation age.

\begin{tabular}{|llll|}
\hline \multirow{2}{*}{ Maternal morbidity } & \multicolumn{2}{l|}{ Gestation age in weeks } & \\
\hline Clinical chorioamnionitis & $\mathbf{< 2 8}$ weeks $(\mathbf{n = 1 0})$ & $\mathbf{2 8 - 3 1}$ weeks $\mathbf{6}$ days $(\mathbf{n = 2 4})$ & $\mathbf{3 2 - 3 6 w e e k s ~ 6}$ days $(\mathbf{n = 7 6})$ \\
\hline Abruptio placentae & $1(10 \%)$ & $2(8.33 \%)$ & $0(0 \%)$ \\
\hline Puerperal pyrexia & $1(10 \%)$ & $1(4.16 \%)$ & $5(6.57 \%)$ \\
\hline Retained placenta & $0(0 \%)$ & $2(8.33 \%)$ & $5(6.57 \%)$ \\
\hline Wound infection & $0(0 \%)$ & $2(8.33 \%)$ & $2(2.63 \%)$ \\
\hline
\end{tabular}

The majority of women $(69.09 \%)$ delivered by LSCS. The indications for caesarean section were intrauterine fetal distress $(26.31 \%)$ precious pregnancy $(23.68 \%)$, severe oligohydramnios and previous caesarean section (15.78\% each). Among the 34 patients who delivered vaginally, $67.64 \%$ had spontaneous delivery, and rest induced by PGE2 gel (8.82\%) and oxytocin $(23.52 \%)$.

In this study, chorioamnionitis was more common in patients who had $>72$ hours of latent period. Puerperal pyrexia $(38.46 \%)$ and abruptio placentae $(30.76 \%)$ were more common among those whose latent period was <24hour latency (Table 3). We also compared the factors causing maternal morbidity with gestational age at PPROM ( $p$ value $<0.001$ ) which is suggestive of clinical chorioamnionitis to be the most common cause of maternal morbidity in patient with gestational age $<28$ weeks (Table 4).

In our study population, $70.2 \%$ had NICU admissions. The mean NICU stay of neonates was $19.77 \pm 17.47$ days who delivered before $<28$ weeks of gestation. While the mean NICU stay in 28-31 weeks 6 days, gestation age group was $6.1 \pm 3.5$ days and 32-36 weeks 6 days gestation age group was $6.03 \pm 5.03$ days). The mean NICU stay was significantly associated with gestational age at birth. ( $\mathrm{p}$ value $<0.001$ )

The perinatal morbidity was $44.21 \%$ with neonatal jaundice $20.89 \%$, respiratory distress syndrome (15.21\%), septicaemia (2.89\%), IVH and pulmonary hypoplasia $(2.17 \%)$, necrotizing colitis $(1.44 \%)$. 
The causes of neonatal morbidity were significantly associated with gestational age at birth ( $\mathrm{p}$ value $<0.001)$. There were 18 neonatal deaths out of which $7.97 \%$ were early preterm babies. In our study, the main cause of perinatal mortality was RDS $(33.33 \%)$ followed by sepsis $(22.22 \%)$ then pulmonary hypoplasia $(16.66 \%)$ and IVH

(16.66\%), necrotizing enterocolitis (11.11) (Table 5).

Table 5: Perinatal outcome in relation to gestation age.

\begin{tabular}{|c|c|c|c|c|}
\hline Neonatal morbidity & $<28$ weeks $(n=14)$ & 28-31 week 6 days $(n=14)$ & 32-36 weeks 6 days $(n=33)$ & \multirow{7}{*}{$\begin{array}{l}\text { P value } \\
<0.001\end{array}$} \\
\hline IVH & $3(21.42 \%)$ & $0(0 \%)$ & $0(0 \%)$ & \\
\hline Necrotizing enterocolitis & $1(7.14 \%)$ & $1(7.14 \%)$ & $0(0 \%)$ & \\
\hline Neonatal Jaundice & $0(0 \%)$ & $3(21.42 \%)$ & $25(75.75 \%)$ & \\
\hline Pulmonary hypoplasia & $2(14.28 \%)$ & $1(7.14 \%)$ & $0(0 \%)$ & \\
\hline RDS & $6(42.85 \%)$ & $7(50 \%)$ & $8(24.24 \%)$ & \\
\hline Sepsis & $2(14.28 \%)$ & $2(14.28 \%$ & $0(0 \%)$ & \\
\hline Total NICU admission & $13(13.40 \%)$ & $28(28.8 \%)$ & $56(57.7 \%)$ & $\begin{array}{l}P \text { value } \\
<0.001\end{array}$ \\
\hline Mean NICU stay & $19.77 \pm 17.47$ days & $6.1 \pm 3.5$ days & $6.03 \pm 5.03$ days & - \\
\hline Neonatal mortality & $11(61.11 \%)$ & $6(33.33 \%)$ & $1(5.55 \%)$ & - \\
\hline
\end{tabular}

\section{DISCUSSION}

Preterm premature rupture of membranes complicates 3$8 \%$ of pregnancies and leads to one third of preterm deliveries. ${ }^{4}$ An incidence of PPROM to be $4.94 \%$. This was comparable to the incidence rate of studies conducted by Tahir et al $(5.4 \%) .^{5}$ The mean age of patients in our study was $30.05 \pm 5.5$ years.

There was no significant correlation between the antenatal care and incidence of PPROM which was in contrast to a study done by Akter et al where there was a strong correlation between the unbooked cases $(90 \%)$ and the incidence of PPROM. ${ }^{6}$ The unbooked cases received poor antenatal care that ultimately leads to increased risk of infection to the mother which is a major risk factor for PPROM.

In our study, 76 cases $(69.09 \%)$ delivered by Lower segment caesarean section. cases. This study is comparable to Singh et al which showed most of cases delivered by $\operatorname{LSCS}(54.36 \%) .^{7}$ Pasquier et al $(58.7 \%)$, Singhal et al (49\%). ${ }^{8,9}$ The caesarean rate was high in our study because our institute is tertiary care referral centre with increased number of IVF conception, bad obstetric history and elderly gravida patients.

In our study the high vaginal swab was positive in $60 \%$ of the cases. E. coli was the most common organism found in cultures, which was present in $36.36 \%$ of PPROM cases, followed by Klebsiella in $18.18 \%$ of cases. We compared the microorganism grown on culture with the gestational age at presentation which came out to be significant (p value 0.040405 ). This shows that $E$. coli is a more common microorganism with advancing gestational age. Similar result obtained with study done by Lovereen et al showed high vaginal swab positive in (22\%) and by Surayapalem et al $(51 \%)$ and the predominant isolated organism from the high vaginal swab in both the studies was Escherichia coli. ${ }^{10,11}$ These culture positive patients were treated with antibiotics according to antibiotic sensitivity test results.

In this study, we compared factors causing maternal morbidity with latent period and the ( $\mathrm{p}$ value) came out 0.21 showing that the relation of maternal morbidity with latent period was non-significant. We also compared the factors causing maternal morbidity with gestational age at PPROM ( $\mathrm{p}$ value $<0.001$ ) which is suggestive of clinical chorioamnionitis to be the most common cause of maternal morbidity in patient with gestational age $<28$ weeks.

Among patients with gestational age 28-31 weeks 6 days there were only $8.33 \%$ cases of clinical chorioamnionitis. No case of chorioamnionitis was found in gestational age 32-36 weeks 6 days. The most common factor for maternal morbidity was abruptio placentae and puerperal pyrexia (6.57\% in both) in gestation age group 32-36 weeks 6 days.

The mean latency period varied inversely with gestational age (Table 1). Out of 110 , two of our patients who presented at 24 weeks of gestation with PPROM were managed conservatively and carried up to 33 weeks. In both the patients, high vaginal swab was positive but with the proper use of antibiotics and corticosteroids, both pregnancies could be prolonged. One patient developed signs of clinical chorioamnionitis and so the pregnancy was terminated by lower segment caesarean section. A Female child $1.4 \mathrm{~kg}$ with Apgar score 9/10 was delivered. The other patient developed severe oligohydramnios and abruptio placenta and so the pregnancy was terminated by lower segment caesarean at 33.2 weeks. A female child $1.6 \mathrm{~kg}$ with Apgar score 9/10 was delivered. None of the patients developed significant maternal morbidity but the conservative management greatly improved neonatal outcome. 
The perinatal morbidity was $40.28 \%$ and neonatal mortality was $13.04 \%$. The main cause of perinatal mortality was RDS $(33.33 \%)$ which is comparable with study by Khade et al in which perinatal morbidity was $33 \%$ and perinatal mortality was $15 \%$ and mainly due to RDS 53\%.12

\section{CONCLUSION}

Management of PPROM revolves around balancing the risk benefit ratio of the conservative treatment. Wherever possible, the treatment should be directed towards conserving the pregnancy with prophylactic use of antibiotics, antenatal corticosteroids and strict vigilance for signs of chorioamnionitis thereby reducing fetal morbidity and mortality without significantly affecting the maternal morbidity. However, termination of pregnancy should be considered at the earliest suspicion of chorioamnionitis. The risk of chorioamnionitis is significantly associated with gestational age at PPROM rather than the duration of latent period. Administration of corticosteroids in all cases reduced the incidence of RDS and IVH. The perinatal mortality can be significantly reduced if we are able to achieve a birth weight of $>1.5 \mathrm{~kg}$. Thus, regular antenatal check-up, planned delivery and dedicated NICU care can significantly reduce the perinatal morbidity and mortality.

Funding: No funding sources

Conflict of interest: None declared

Ethical approval: The study was approved by the Institutional Ethics Committee

\section{REFERENCES}

1. Mercer BM. Preterm premature rupture of the membranes. Obstet Gynecol. 2003;101(1):178-93.

2. Caughey AB, Robinson JN, Norwitz ER. Contemporary diagnosis and management of preterm premature rupture of membranes. Rev Obstet Gynecol. 2008;1(1):11-22.

3. Morris JM, Roberts CL, Crowther CA, Buchanan SL, Henderson-Smart DJ, Salkeld G. Protocol for the immediate delivery versus expectant care of woman with preterm prelabour rupture of membranes close to term (PPROM) trial. BMC Pregnanc Childbirth 2006;6:9.

4. Arias F. Premature rupture of the membrane. In: Practical guide to high risk pregnancy and delivery. 3rd edn. Elsevier: New Delhi; 2008:220-237.

5. Tahir S, Aleem M, Aziz R. Incidence and outcome of preterm-premature rupture of membranes. Pak J Med Sci. 2002;18(1):26-32.

6. Akter S, Akter R, Rashid M. Preterm prelabour rupture of the membrane and feto-maternal outcome: an observational study. J Bangladesh Coll Phys Surg. 2010;28(1):17-23.

7. Singh J, Kanti V, Verma V. Study of fetomaternal outcome in cases of premature rupture of membrane at tertiary care rural institute of Western Uttar Pradesh, India. Int $\mathbf{J}$ Reprod Contracept Obstet Gynecol. 2020;9(1):78.

8. Pasquier JC, Rabilloud M, Picaud JC, Ecochard R, Claris O, Gaucherand $\mathrm{P}$, et al. A prospective population-based study of 598 cases of PPROM between 24- and 34-weeks' gestation: description, management, and mortality (DOMINOS cohort). Eur J Obstet Gynecol Reprod Biol. 2005;121(2):164-70.

9. Singhal P, Sharma S, Garg R. Perinatal mortality in caesarean section. J Obstet Gynec India. 1994;44:751-6.

10. Lovereen S, Khanum MA, Nargis N, Begum S, Afroze R. Maternal and neonatal outcome in premature rupture of membranes. Bangladesh J Med Sci. 2018;17(3):479-83.

11. Surayapalem S, Cooly V, Salicheemala B. A study on maternal and perinatal outcome in premature rupture of membranes at term. Int $\mathbf{J}$ Reprod Contracept Obstet Gynecol. 2017;6(12):5368.

12. Khade SA, Bava AK. Preterm premature rupture of membranes: maternal and perinatal outcome Int $\mathbf{J}$ Reprod Contracept Obstet Gynecol. 2018;7(11):4499-505.

Cite this article as: Chundawat R, Singh M, Gupta A, Jakhetiya B, Khan R. Fetomaternal outcomes in conservative management of preterm premature rupture of membrane. Int J Reprod Contracept Obstet Gynecol 2021;10:691-5. 